\title{
An improving method for micro-G simulation with magnetism-buoyancy hybrid system
}

\author{
Zhanxia Zhu ${ }^{1,2, * 1}$,Jianping Yuan ${ }^{1,2, *}$, Jiangzhou Song ${ }^{1,2}, \quad$ Rongxin Cui ${ }^{1}$ \\ ${ }^{1}$ Northwestern Polytechnical University, 127 West Youyi Road, Xi'an, 710072, PR China \\ ${ }^{2}$ National Key Laboratory of Aerospace Flight Dynamics, 127 West Youyi Road, Xi'an, 710072, PR China
}

\begin{abstract}
This paper presents a novel solution for the micro-G experiment with magnetism-buoyancy hybrid system. The improvement includes two parts, (i) proposing an innovative system called general balance test bed (GBTB), and (ii) designing a resistance effect compensation system. The GBTB, a special platform, can be used to realize the effect of neutral buoyancy, by using controllable electromagnetic force instead of conventional weight or foam module to eliminate the difference between gravity and liquid buoyancy. In this paper, principles, components, and functions of the GBTB are developed. Then, in order to improve test fidelity, a compensation system is designed to counteract the water resistance effect during maneuver, and a novel prediction law is proposed to make water resistance force prediction more coincident with the real value by introducing control errors and error rates. Finally, the feasibility and effectiveness of the proposed solution are demonstrated through micro-G experiments and tests.
\end{abstract}

Keywords: magnetism-buoyancy hybrid system; micro-G simulation; resistance effect compensation; general balance test bed

\section{Introduction}

As is well known, all the new discoveries must be tested before application, and this is even more rigorous to future complex space operations. The ground test and demonstration on corresponding theories and technologies require that experimental system should provide a long-duration, large-scale, highly-accurate, controllable and almost real microgravity test environment to simulate the space motion in the same dimension as in space.

Traditional ground micro-G experimental facilities can't fully meet the above requirements because of their insufficiencies ${ }^{[1]}$. Among these facilities based on traditional methods (such as zero-G airplane, drop tower, air-bearing suspension system and neutral buoyancy system), the neutral buoyancy system (NBS) seems to be most suitable for verifying complex space operations because of its large volume, long-duration and 6 degree-of-freedom (DOF) space.

Several organizations in the world own their neutral buoyancy tank, but it is mainly used to train astronauts and is not widely applied to testing complex space operations. Papers in open literature show that only the Space Systems Laboratory (SSL) at the University of Maryland and MIT use neutral buoyancy facilities to study complex space operations. For example, the SSL successfully developed many types of robots ${ }^{[2-6]}$ to verify autonomous rendezvous and docking, in-orbit assembly, inspection and maintenance technologies. MIT performed several tests of a teleoperation manipulator using NASA's neutral buoyancy lab (NBL) ${ }^{[6]}$. Besides, it is almost no other organization using neutral

\footnotetext{
* Corresponding author at: School of Astronautics, Northwestern Polytechnical University, Xi'an, 710072, PR China.

E-mail addresses: zhuzhanxia@nwpu.edu.cn (Z.Zhu), jyuan@nwpu.edu.cn (J. Yuan).
} 
buoyancy tank to do such experiments. The causes may be: 1) Uncontrollable, the simulation accuracy is influenced by the balancing results, which are difficult to be adjusted and controlled online with environmental force. 2) Non-consistency, there is no common balancing system suitable for different test body, thus we have to develop new balancing system for a new test body, and it is a complex process. 3) Low-fidelity, the neutral buoyancy system suffers from the water resistance force induced by water which, however, does not exit in space, thus the test fidelity is low.

The first issue has been solved in reference ${ }^{[1]}$ by using magnetism-buoyancy hybrid micro-G simulation system (MBHMSS), but the other two problems yet exist. For the non-consistency of balancing system, we propose and develop a general balance test bed (GBTB) in MBHMSS, with which we can eliminate the difference between gravity and liquid buoyancy (the difference is called as residual gravity in this paper) on new test body quickly with controllable electromagnetic force, not only make the simulation accuracy controllable in real time but also simplify the balancing process. In order to improve the fidelity of system, we design a compensation control law based on water resistance force prediction, in which a novel prediction algorithm is proposed to predict the water resistance force exactly by introducing control errors and error rates. With above methods, the drawbacks of the neutral buoyancy system can be overcome, while the advantages can be retained.

\section{General balance test bed}

A micro-G experiment needs a free-floating test body under water to keep a neutral buoyancy state in the whole experimental space. It includes two aspects: 1) balance between gravity and buoyancy (known as the balance of force in its vertical direction); 2) the center of gravity and the center of buoyancy are coincident (also called rotational neutral balance). In general, adding weight or foam module to the test body can achieve neutral buoyancy, but the shortcomings of this method are: one can only add clump weight or buoyancy module to some particular points on the surface of the test body; thus it is difficult to implement both force balance and rotational neutral balance at the same time. In addition, the method requires trained divers to perform underwater balance and has to be repeatedly used many times to obtain a high balance effect, which is largely influenced by a diver's skills and experience and needs to take a long time to complete. In order to overcome these shortcomings, J.D. Graves ${ }^{[4]}$ put forward a kind of neutral buoyancy balance system, which is installed in the test body and includes a coarse balance system, an accurate gravity and buoyancy balance system and a rotational neutral balance system. This neutral buoyancy balance system, in our opinion, is a relatively effective method, but the coarse balancing process still needs to be tested out of water and underwater for many times. This complicated process takes a long time, and the test body should be tested again once it changes. Also, the structural and mass characteristics of the test body are not similar to its prototype.

We propose a novel method to quickly achieve neutral buoyancy balance by changing the environmental force on the test body. The rotational neutral balance should be achieved by the design of the test body, i.e., making its gravity center coincide with its buoyancy center. Usually, the gravity and buoyancy of a test body are different, and the difference is defined as residual gravity. The neutral buoyancy can be achieved by ensuring that the residual gravity is zero. We introduce a controllable electromagnetic force to compensate for the residual gravity ${ }^{[1]}$. As shown in Fig. 1, the balance of electromagnetic force in its vertical direction and the residual gravity is achieved by placing the test body in electromagnetic field, generating the electromagnetic force and controlling it. Automatic control may simplify and quicken this process, and it usually takes a few minutes to achieve the 
balance.

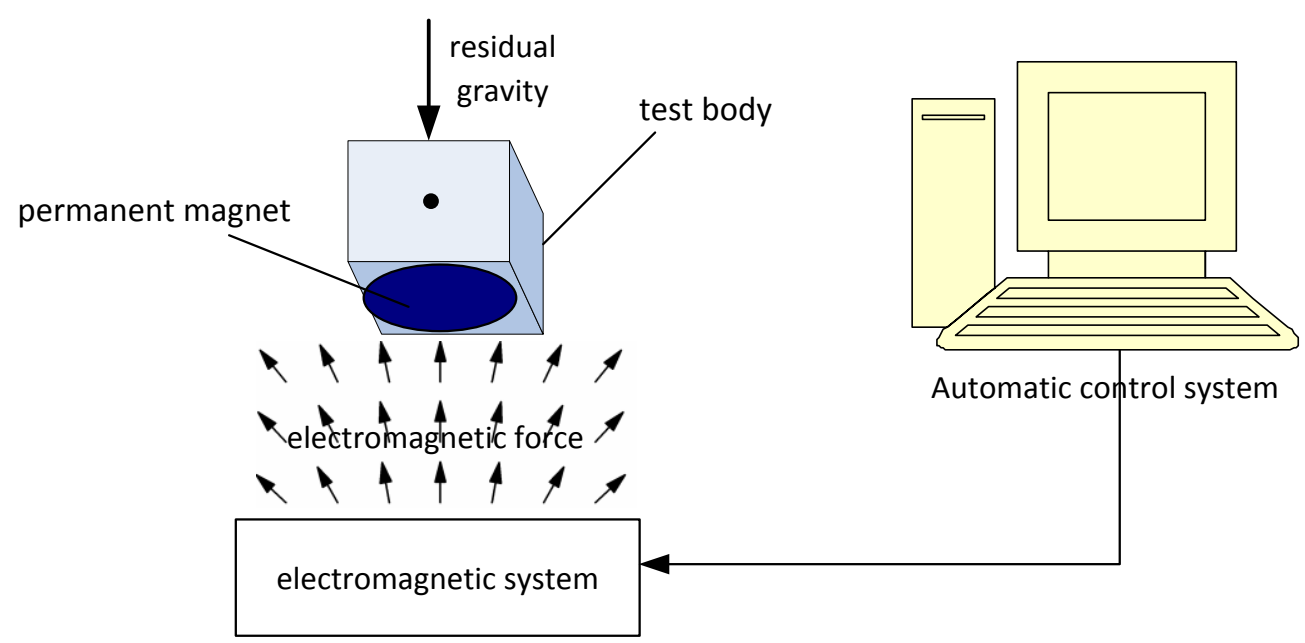

Fig. 1. The schematic diagram for the residual gravity balance.

For a given test body, the residual gravity is definite, so the electromagnetic force should be constant in its vertical direction. But electromagnetic force is a space vector and is unable to meet this requirement. Therefore, we build the distributed electromagnetic system with the vector superposition equivalent and homogeneous magnetic field, a permanent magnet is linked to the test body and multiple electromagnetic forces act on the permanent magnet, if the electromagnetic field is symmetrical, the resultant electromagnetic force will be in vertical direction and constant by controlling ${ }^{[1]}$. When the permanent magnet has non-zero attitude angle related to a horizontal plane or is not at the center of the distributed electromagnetic system, the electromagnetic field will not be symmetrical, and the direction of electromagnetic force will not be along the vertical direction. So the symmetrical system means that the permanent magnet should be strictly symmetrical and kept horizontal at the center of the distributed magnetic system. Because the permanent magnet is linked to the test body, so we wish: 1) its attitude should not be affected by the test body; 2) the electromagnetic force should be acted to the test body. Therefore, how to link the permanent magnet with the test body is a key to obtaining the constant electromagnetic force.

We propose a general balance test bed (GBTB), which is composed of basement and framework system, as shown in Fig. 2. The basement of the GBTB is generally cylindrical in order to reduce motion resistance, and the permanent magnet is fixed inside. The framework system is designed to have two DOFs according to the theory of stable gyros with two axes. The outer framework is joined with the basement, while the inner axle is fixed to the test body. Hence, the attitude of the test body is decoupled with GBTB. When the permanent magnet is installed at the bottom of the basement, the center of gravity is located below the center of buoyancy for GBTB. If AB is the centerline of GBTB, the mass center and the buoyancy center will be located at the $A B$ because of its symmetry. Thus the attitude of the permanent magnet will be kept horizontal easily. 


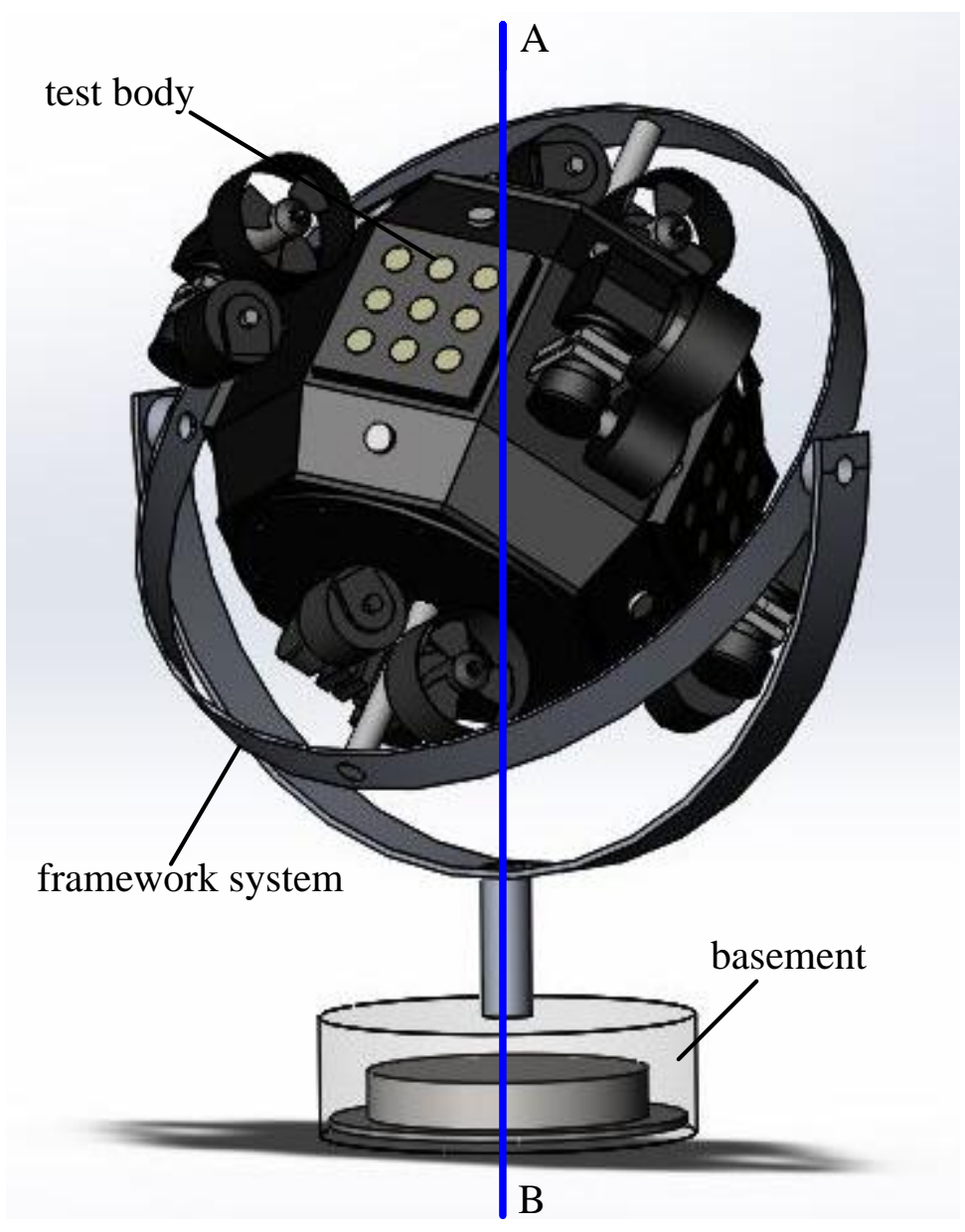

Fig. 2. The schematic diagram for GBTB.

Usually GBTB is demanded to have micro-G in water, which can be achieved by selecting a proper size of the basement, which is expressed as:

$$
V_{c}=\left(m_{c}+m_{\mathrm{p}}+m_{f}\right) \times 10^{-3}-V_{f}
$$

where $V_{c}$ is the size of cylinder; $V_{f}$ is the size of the framework system; $m_{c}$ is the mass of the basement; $m_{\mathrm{p}}$ is the mass of the permanent magnet; $m_{f}$ is the mass of the framework system;

When we take the water density as $1.0 \times 10^{3} \mathrm{~kg} / \mathrm{m}^{3}$, then $10^{-3}$ appears in the right side of above equation. If the diameter of the cylinder is the same as the size of the outer framework system, then the outline of the cylinder can be obtained.

If GBTB and test body are treated as a whole system, its mass center and float center in the test body's coordinate frame will not change, whatever the attitude of the test body changes. We place a main booster on the mass center to control the position of the test body and to simulate its orbit motion control. We also install an attitude booster at the position similar to that of the space prototype to simulate its attitude control. Thus we can perform the 6-DOFs motion test on three positions and three attitudes with these facilities. 
Unlike the traditional neutral buoyancy balancing method with adding weight or foam module, it can not be used to get promising consistency for a new test body or repeated tests. With GBTB, for a given test body, one can repeat the same test many times without worrying about the condition of the test body changed, such as the mass characteristics of the test body, because no mass would be add to the system, thus the consistency of repeated results will be maintained. The GBTB is also a common platform system, it can carry different test bodies with different residual gravity and different mass and different profile, for a new test body, it can be placed on the GBTB and balance the residual gravity quickly and easily by controllable electromagnetic force, and the micro-G level can also be adjusted in real time as expected.

\section{Water resistance effect compensation with motion feedback and prediction}

When moving in water, the test body suffers from water resistance force induced by viscosity inevitably. For NBS and MBHMSS, water resistance force will make test fidelity low. In order to obtain similar experiment system and improve test fidelity, the effect of water resistance force must be eliminated. If the water resistance force can be balanced with active thrust force, their effects on test body will offset. Usually, the active thrust force is also used to control the motion of test body. Then the control instruction fed into the actuating mechanism is the superposition of the compensation control quantity and the motion control quantity. Ideally, if both two control quantities are generated by different controllers, they will be uncoupled, then one can analysis the results of compensation and motion control separately. Thus, the test is not only helpful for analyzing the equivalence between ground experiment control instruments and real spatial experiment instruments, but also directly verify the validity and feasibility of the spatial control strategy in the ground experimental environment.

One objective of this paper is to compensate the water resistance force without considering the mission control. The active thrust force is used to balance the water resistance force, which is predictable according to the configuration of the test body and the desired motion parameters (ideal state). When thrust force with the same size as the water resistance force but in an opposite direction, the resistance compensation can be realized, and the motion characteristic of test body in ground system is the same as it in space. But actually, they are not equivalence in size because of control error, the real motion parameters are not equal to its desired, so the real water resistance force is different from the prediction value, the compensation error is arise accordingly. Therefore, we design a compensation control law by combining the water resistance force prediction with error control, and a novel prediction algorithm is proposed by introducing control error and error rates to predict the water resistance force exactly. The design procedure is described in detail as follows.

\subsection{The motion equations of the test body}

In order to build the motion equations, we choose the geographic coordinate system $o x_{n} y_{n} z_{n}$ as the inertial coordinate system, in which $o x_{n}$ points to the local north, $o y_{n}$ points to the local east and $o z_{n}$ points to the earth's core. $o x_{b} y_{b} z_{b}$ is defined as the test body's coordinate system, in which $o x_{b}$ is along the longitudinal axis pointing forward, $o z_{b}$ is located in vertical plane and straights 
down, $o y_{b}$ is perpendicular to the surface $o x_{b} z_{b}$ and constitutes a right-handed coordinate frame.

Hence, the 6-DOFs equations of the test body in the test body's coordinate system $o x_{b} y_{b} z_{b}$ are described as Eq. (1):

$$
\left\{\begin{array}{r}
M \dot{v}+C(v) v+D(v) v+g(\eta)=\tau_{c} \\
\dot{\eta}=J(\eta) v
\end{array}\right.
$$

where $\boldsymbol{v}$ is the generalized velocity of the test body and $v=[u, v, w, p, q, r]^{T}, u, v, w$ are the projection of linear velocity vector in $o x_{b} y_{b} z_{b}$ and $p, q, r$ are the projection of angular velocity vector in ${ } x_{b} y_{b} z_{b}$. Matrix $\boldsymbol{M}$ is the inertia mass matrix of the test body; if $\mathrm{m}$ is the mass of the test body, $\boldsymbol{r}_{G}=\left[x_{G}, y_{G}, z_{G}\right]^{T}$ is the position of mass center, let $\boldsymbol{I}_{0}=\left[\begin{array}{ccc}I_{x x} & -I_{x y} & -I_{x z} \\ -I_{y x} & I_{y y} & -I_{y z} \\ -I_{z x} & -I_{z y} & I_{z z}\end{array}\right]$ $\boldsymbol{M}=\left[\begin{array}{llllll}m & 0 & 0 & 0 & m z_{G} & -m y_{G} \\ 0 & m & 0 & -m z_{G} & 0 & m x_{G} \\ 0 & 0 & m & m y_{G} & -m x_{G} & 0 \\ 0 & -m z_{G} & m y_{G} & I_{x x} & -I_{x y} & -I_{x z} \\ m z_{G} & 0 & -m x_{G} & -I_{y x} & I_{y y} & -I_{y z} \\ -m y_{G} & m x_{G} & 0 & -I_{z x} & -I_{z y} & I_{z z}\end{array}\right]$

$\boldsymbol{C}(\boldsymbol{v})$ is the Coriolis force matrix of the test body, which can be obtained with the inertia matrix parameters of test body, using the following equation:

$$
\boldsymbol{C}(\boldsymbol{v})=\left[\begin{array}{cc}
m \mathrm{~S}\left(\boldsymbol{\omega}_{b}\right) & -m \mathrm{~S}\left(\boldsymbol{\omega}_{b}\right) \mathrm{S}\left(\boldsymbol{r}_{G}\right) \\
m \mathrm{~S}\left(\boldsymbol{r}_{G}\right) \mathrm{S}\left(\boldsymbol{\omega}_{b}\right) & -\mathrm{S}\left(\boldsymbol{I}_{0} \boldsymbol{\omega}_{b}\right)
\end{array}\right]
$$

where $\boldsymbol{\omega}_{b}=[p, q, r]^{T}$ is the angular velocity vector of the test body; $S(\square)$ is the vector operator. If the vector $\boldsymbol{a}=\left[\begin{array}{lll}a_{1} & a_{2} & a_{3}\end{array}\right]^{T} \in \boldsymbol{R}^{3}, \boldsymbol{b}=\left[\begin{array}{lll}b_{1} & b_{2} & b_{3}\end{array}\right]^{T} \in \boldsymbol{R}^{3}$, then $\boldsymbol{a} \times \boldsymbol{b}=S(\boldsymbol{a}) \boldsymbol{b}$ and $S(\boldsymbol{a})=-S^{T}(\boldsymbol{a})=\left[\begin{array}{ccc}0 & -a_{3} & a_{2} \\ a_{3} & 0 & -a_{1} \\ -a_{2} & a_{1} & 0\end{array}\right]$

$\boldsymbol{D}(\boldsymbol{v})$ is a diagonal, damping matrix, usually $\boldsymbol{D}(\boldsymbol{v})=-\operatorname{diag}\left\{X_{u|u|}, Y_{v|v|}, Z_{w|w|}, K_{p|p|}, M_{q|q|}, N_{r|r|}\right\}$, in which a diagonal element is the coefficient of hydrodynamic force. For a given test body, it can be obtained by evaluation or test. $\boldsymbol{g}(\boldsymbol{\eta})$ is the negative buoyancy vector, which is decided by the size of neutral buoyancy. The elements of $\boldsymbol{g}(\boldsymbol{\eta})$ can be obtained from the gravity vector $\boldsymbol{W}$, buoyancy 
vector $\boldsymbol{B}$, roll angle $\phi$ and pitch angle $\theta$ as described in Eq. (5). Here, $\boldsymbol{\eta}=\left[\begin{array}{c}\boldsymbol{p} \\ \boldsymbol{\Theta}\end{array}\right]$ and $\boldsymbol{p}=\left[\begin{array}{lll}x & y & z\end{array}\right]^{T}$ are the position of the test body described in $o x_{n} y_{n} z_{n} ; \quad \Theta=\left[\begin{array}{lll}\phi & \theta & \psi\end{array}\right]^{T}$ is the Eular angle (ZYX order) that consists of roll angle $\phi$, yaw angle $\psi$ and pitch angle $\theta$.

$$
\boldsymbol{g}(\boldsymbol{\eta})=-\left[\begin{array}{c}
-(W-B) \sin \theta \\
(W-B) \cos \theta \sin \phi \\
(W-B) \cos \theta \cos \phi \\
-\left(y_{G} W-y_{B} B\right) \cos \theta \cos \phi-\left(z_{G} W-z_{B} B\right) \cos \theta \sin \phi \\
-\left(z_{G} W-z_{B} B\right) \sin \theta-\left(x_{G} W-x_{B} B\right) \cos \theta \cos \phi \\
-\left(x_{G} W-x_{B} B\right) \cos \theta \sin \phi-\left(y_{G} W-y_{B} B\right) \sin \theta
\end{array}\right]
$$

where $W$ is the value of gravity, $B$ is the value of buoyancy, $\boldsymbol{r}_{B}=\left[x_{B}, y_{B}, z_{B}\right]^{T}$ is the position of the float center. If the gravity is equal to the buoyancy and the mass center coincides with the float center absolutely leads to $\boldsymbol{g}(\boldsymbol{\eta})=0 . \boldsymbol{J}(\boldsymbol{\eta})$ is the kinetic coefficient matrix

$$
\boldsymbol{J}(\boldsymbol{\eta})=\left[\begin{array}{cc}
\boldsymbol{R}_{b}^{n}(\boldsymbol{\Theta}) & \boldsymbol{0}_{3 \times 3} \\
\boldsymbol{0}_{3 \times 3} & \boldsymbol{T}_{\boldsymbol{\Theta}}(\boldsymbol{\Theta})
\end{array}\right]
$$

where $\quad \boldsymbol{R}_{n}^{b}(\boldsymbol{\Theta})=\left[\begin{array}{ccc}\cos \theta \cos \psi & \cos \theta \sin \psi & -\sin \theta \\ \sin \varphi \sin \theta \cos \psi-\cos \varphi \sin \psi & \cos \varphi \cos \psi+\sin \varphi \sin \theta \sin \psi & \sin \varphi \cos \theta \\ \sin \varphi \sin \psi+\cos \varphi \cos \psi \sin \theta & \cos \varphi \sin \theta \sin \psi-\sin \varphi \cos \psi & \cos \varphi \cos \theta\end{array}\right]$ and $\boldsymbol{T}_{\boldsymbol{\Theta}}(\boldsymbol{\Theta})=\left[\begin{array}{ccc}1 & \sin \varphi \tan \theta & \cos \varphi \tan \theta \\ 0 & \cos \varphi & -\sin \varphi \\ 0 & \sin \varphi / \cos \theta & \cos \varphi / \cos \theta\end{array}\right] \cdot \boldsymbol{\tau}_{c}$ is the external control input of the test body.

\subsection{The compensation control law with water resistance force prediction and error control}

Ideally, we hope the resultant force from the environment upon the test body is zero during the experiment. According to Newton's second law, Eq. (1) can be written as:

$\boldsymbol{M} \dot{\boldsymbol{v}}=\boldsymbol{\tau}$

where $\boldsymbol{\tau}$ is the external force exerted on the test body, $\boldsymbol{\tau}=\boldsymbol{\tau}_{c}-\boldsymbol{\tau}_{f}, \boldsymbol{\tau}_{c}$ is the control force, and $\boldsymbol{\tau}_{f}$ is the compensation force predicted with the following equation:

$\tau_{f}=C(v) v+D(v) v+g(\eta)$

$\boldsymbol{\tau}_{f}$ is related to $\boldsymbol{v}$ and can be evaluated with measurement parameters. 
The dynamic equation of the test body can be expressed in $o x_{n} y_{n} z_{n}$ by using the transformation between $o x_{n} y_{n} z_{n}$ and $o x_{b} y_{b} z_{b}$ as follows:

$\boldsymbol{M}_{\eta}(\boldsymbol{\eta}) \ddot{\boldsymbol{\eta}}+\boldsymbol{C}_{\eta}(\boldsymbol{v}, \boldsymbol{\eta}) \dot{\boldsymbol{\eta}}+\boldsymbol{D}_{\eta}(\boldsymbol{v}, \boldsymbol{\eta}) \dot{\boldsymbol{\eta}}+\boldsymbol{g}_{\eta}(\boldsymbol{\eta})=\boldsymbol{J}^{-\mathrm{T}}(\boldsymbol{\eta}) \boldsymbol{\tau}$

where $\boldsymbol{M}_{\eta}(\boldsymbol{\eta})=\boldsymbol{J}^{-\mathrm{T}}(\boldsymbol{\eta}) \boldsymbol{M J}^{-1}(\boldsymbol{\eta})$, and $\boldsymbol{M}_{\boldsymbol{\eta}}(\boldsymbol{\eta})=\boldsymbol{M}_{\boldsymbol{\eta}}{ }^{T}(\boldsymbol{\eta})>0$.

$\boldsymbol{C}_{\eta}(\boldsymbol{v}, \boldsymbol{\eta})=\boldsymbol{J}^{-\mathrm{T}}(\boldsymbol{\eta})\left[\boldsymbol{C}(\boldsymbol{v})-\boldsymbol{M J}^{-1}(\boldsymbol{\eta}) \dot{\boldsymbol{J}}(\boldsymbol{\eta})\right] \boldsymbol{J}^{-1}(\boldsymbol{\eta})$, and $\boldsymbol{C}_{\eta}(\boldsymbol{v}, \eta) \neq-\boldsymbol{C}_{\eta}^{\mathrm{T}}(\boldsymbol{v}, \eta)$.

$\boldsymbol{D}_{\eta}(\boldsymbol{v}, \boldsymbol{\eta})=\boldsymbol{J}^{-\mathrm{T}}(\boldsymbol{\eta}) \boldsymbol{D}(\boldsymbol{v}) \boldsymbol{J}^{-1}(\boldsymbol{\eta})$, and $\boldsymbol{D}_{\eta}(\boldsymbol{v}, \boldsymbol{\eta})>0 . \quad \boldsymbol{g}_{\eta}(\boldsymbol{\eta})=\boldsymbol{J}^{-\mathrm{T}}(\boldsymbol{\eta}) \boldsymbol{g}(\boldsymbol{\eta}), \quad \boldsymbol{v}=\boldsymbol{J}^{-1}(\boldsymbol{\eta}) \dot{\boldsymbol{\eta}}$,

$\dot{\boldsymbol{v}}=\boldsymbol{J}^{-1}(\boldsymbol{\eta})\left[\ddot{\boldsymbol{\eta}}-\dot{\boldsymbol{J}}(\boldsymbol{\eta}) \boldsymbol{J}^{-1}(\boldsymbol{\eta}) \dot{\boldsymbol{\eta}}\right]$.

For $\boldsymbol{\tau}_{f}=\boldsymbol{C}(\boldsymbol{v}) \boldsymbol{v}+\boldsymbol{D}(\boldsymbol{v}) \boldsymbol{v}+\boldsymbol{g}(\boldsymbol{\eta})$, we can choose a set of parameters to make $\boldsymbol{C}(\boldsymbol{v}), \boldsymbol{D}(\boldsymbol{v}), \boldsymbol{g}(\boldsymbol{\eta})$ satisfy the linear relationship as described in Eq. (10):

$C(v) v+D(v) v+g(\eta) \square Y(\dot{v}, v, \eta) \lambda$

where $\boldsymbol{Y}(\dot{\boldsymbol{v}}, \boldsymbol{v}, \boldsymbol{\eta})$ is known parameters matrix of the system, $\lambda$ is a vector described with an unknown physical quantity of the test body ${ }^{[7]}$.

Based on the dynamic model as given in Eq. (9), a compensation control law can be designed to compensate $\boldsymbol{\tau}_{f}$ entirely, which makes the real states follow the desired ideal states exactly. We estimate $\boldsymbol{\tau}_{f}$ according to the real states and meanwhile consider the compensation control errors and error rates (induced by the performance of the actuator). Therefore, we combine the prediction of $\boldsymbol{\tau}_{f}$ with control errors to make $\boldsymbol{\tau}_{f}$ more coincident with the real value. In this paper, we design an adaptive prediction method with PD controller.

Define

$\tilde{\boldsymbol{\eta}}=\boldsymbol{\eta}-\boldsymbol{\eta}_{d}$

$\dot{\tilde{\boldsymbol{\eta}}}=\dot{\boldsymbol{\eta}}-\dot{\boldsymbol{\eta}}_{d}$

where $\boldsymbol{\eta}$ is the real state of the test body. $\boldsymbol{\eta}_{d}$ is the desired ideal state of the test body in $o x_{n} y_{n} z_{n}$, $\tilde{\boldsymbol{\eta}}$ is the error which is the different between real state and desired ideal state at any time.

We introduce a new parameter $s$ to describe the control result, which includes error and its differential, as expressed in the following:

$\boldsymbol{s}=\dot{\tilde{\boldsymbol{\eta}}}+\Lambda \tilde{\boldsymbol{\eta}}$

where $\boldsymbol{\Lambda}$ is a constant positive symmetrical matrix.

Based on Eq. (10), the prediction of $\boldsymbol{\tau}_{f}$ can be expressed as follows:

$\hat{\boldsymbol{\tau}}_{f}=\boldsymbol{Y}(\dot{\boldsymbol{v}}, \boldsymbol{v}, \boldsymbol{\eta}) \hat{\lambda}$

where $\hat{\boldsymbol{\tau}}_{f}$ is the evaluation of $\boldsymbol{\tau}_{f}, \hat{\lambda}$ is the evaluation of $\lambda$.

In order to obtain $\hat{\boldsymbol{\tau}}_{f}$, we design an prediction law to obtain the value of $\hat{\lambda}$ by using parameter $s$, as shown in Eq. (15):

$\dot{\hat{\lambda}}=-\boldsymbol{\Gamma}^{-1} \boldsymbol{Y}^{\mathrm{T}}(\dot{\boldsymbol{v}}, \boldsymbol{v}, \boldsymbol{\eta}) \boldsymbol{Y}(\dot{\boldsymbol{v}}, \boldsymbol{v}, \boldsymbol{\eta}) \boldsymbol{J}^{-1}(\boldsymbol{\eta}) \boldsymbol{s}$

where $\boldsymbol{\Gamma}$ is a positive symmetrical matrix, $\boldsymbol{\Gamma}=\boldsymbol{\Gamma}^{T}>\boldsymbol{0}$. 
Based on the water resistance force prediction and error control, we design a compensation control law as follows:

$$
\boldsymbol{\tau}=\boldsymbol{Y}(\dot{\boldsymbol{v}}, \boldsymbol{v}, \boldsymbol{\eta}) \hat{\boldsymbol{\lambda}}-\boldsymbol{J}^{\mathrm{T}}(\boldsymbol{\eta}) \boldsymbol{K}_{d} \boldsymbol{s}
$$

where, the first term is the water resistance force compensation, the second term is error control based on PD feedback control, $\boldsymbol{K}_{d}$ is the feedback gain and $\boldsymbol{K}_{d}$ is a positive symmetrical matrix.

In order to prove the stability of the above compensation control law, we choose the following Lyapunov function:

$$
V(t)=\frac{1}{2} \boldsymbol{s}^{T} \boldsymbol{M}_{\eta} \boldsymbol{s}+\frac{1}{2} \tilde{\lambda}^{T} \boldsymbol{\Gamma} \tilde{\boldsymbol{\lambda}}
$$

where $\tilde{\lambda}=\hat{\lambda}(t)-\lambda$. The derivative of $V(t)$ is

$$
\begin{aligned}
\dot{V}(t) & =\frac{1}{2}\left(\boldsymbol{s}^{T} \boldsymbol{M}_{\eta} \dot{\boldsymbol{s}}+\dot{\boldsymbol{s}}^{T} \boldsymbol{M}_{\eta} \boldsymbol{s}+\boldsymbol{s}^{T} \dot{\boldsymbol{M}}_{\eta} \boldsymbol{s}\right)+\frac{1}{2}\left(\dot{\tilde{\lambda}}^{T} \boldsymbol{\Gamma} \tilde{\boldsymbol{\lambda}}+\tilde{\lambda}^{T} \boldsymbol{\Gamma} \dot{\tilde{\lambda}}\right) \\
& =\frac{1}{2}\left(\boldsymbol{s}^{T} \boldsymbol{M}_{\eta} \dot{\boldsymbol{s}}+\dot{\boldsymbol{s}}^{T} \boldsymbol{M}_{\eta} \boldsymbol{s}+\boldsymbol{s}^{T} \dot{\boldsymbol{M}}_{\eta} \boldsymbol{s}\right)+\dot{\tilde{\lambda}}^{T} \boldsymbol{\Gamma} \tilde{\boldsymbol{\lambda}} \\
& =\frac{1}{2} \boldsymbol{s}^{T} \dot{\boldsymbol{M}}_{\eta} \boldsymbol{s}+\boldsymbol{s}^{T} \boldsymbol{M}_{\eta} \dot{\boldsymbol{s}}+\dot{\tilde{\lambda}}^{T} \boldsymbol{\Gamma} \tilde{\boldsymbol{\lambda}}
\end{aligned}
$$

If we substitute $s=\dot{\tilde{\boldsymbol{\eta}}}+\boldsymbol{\Lambda} \tilde{\boldsymbol{\eta}}$ into Eq. (18), then the following is obtained:

$$
\begin{aligned}
\dot{V}(t) & =\frac{1}{2} \boldsymbol{s}^{T} \dot{\boldsymbol{M}}_{\eta} \boldsymbol{s}+\boldsymbol{s}^{T} \boldsymbol{M}_{\eta} \dot{\boldsymbol{s}}+\dot{\tilde{\boldsymbol{\lambda}}}^{T} \boldsymbol{\Gamma} \tilde{\boldsymbol{\lambda}} \\
& =\frac{1}{2} \boldsymbol{s}^{T} \dot{\boldsymbol{M}}_{\eta} \boldsymbol{s}+\boldsymbol{s}^{T} \boldsymbol{M}_{\eta}(\ddot{\tilde{\boldsymbol{\eta}}}+\boldsymbol{\Lambda} \dot{\tilde{\boldsymbol{\eta}}})+\dot{\tilde{\lambda}}^{T} \boldsymbol{\Gamma} \tilde{\boldsymbol{\lambda}} \\
& =-\boldsymbol{s}^{T} \boldsymbol{D}_{\eta} \boldsymbol{s}+\boldsymbol{s}^{T}\left(\boldsymbol{J}^{-\mathrm{T}} \boldsymbol{\tau}-\boldsymbol{M}_{\eta} \ddot{\boldsymbol{\eta}}_{r}-\boldsymbol{C}_{\eta} \dot{\boldsymbol{\eta}}_{r}-\boldsymbol{D}_{\eta} \dot{\boldsymbol{\eta}}_{r}-\boldsymbol{g}_{\eta}\right)+\dot{\tilde{\lambda}}^{T} \boldsymbol{\Gamma} \tilde{\lambda}
\end{aligned}
$$

If we substitute Eqs. (15) and (16) into Eq. (19), then the following is obtained:

$$
\begin{aligned}
\dot{V}(t) & =-\boldsymbol{s}^{T} \boldsymbol{D}_{\eta} \boldsymbol{s}+\boldsymbol{s}^{T} \boldsymbol{J}^{-\mathrm{T}}(\boldsymbol{\eta})\left[\boldsymbol{Y}(\dot{\boldsymbol{v}}, \boldsymbol{v}, \boldsymbol{\eta}) \hat{\boldsymbol{\lambda}}-\boldsymbol{J}^{\mathrm{T}}(\boldsymbol{\eta}) \boldsymbol{K}_{d} \boldsymbol{s}-\boldsymbol{Y}(\dot{\boldsymbol{v}}, \boldsymbol{v}, \boldsymbol{\eta}) \lambda\right]+\dot{\tilde{\lambda}}^{T} \boldsymbol{\Gamma} \tilde{\boldsymbol{\lambda}} \\
& =-\boldsymbol{s}^{T}\left(\boldsymbol{K}_{d}+\boldsymbol{D}_{\eta}\right) \boldsymbol{s}+\tilde{\boldsymbol{\lambda}}^{T} \dot{\boldsymbol{\Gamma}}+\left[\boldsymbol{J}^{-1}(\boldsymbol{\eta}) \boldsymbol{s}\right]^{\mathrm{T}} \boldsymbol{Y}(\dot{\boldsymbol{v}}, \boldsymbol{v}, \boldsymbol{\eta}) \tilde{\boldsymbol{\lambda}} \\
& =-\boldsymbol{s}^{T}\left(\boldsymbol{K}_{d}+\boldsymbol{D}_{\eta}\right) \boldsymbol{s}+\tilde{\boldsymbol{\lambda}}^{T} \boldsymbol{Y}^{\mathrm{T}}(\dot{\boldsymbol{v}}, \boldsymbol{v}, \boldsymbol{\eta}) \boldsymbol{Y}(\dot{\boldsymbol{v}}, \boldsymbol{v}, \boldsymbol{\eta}) \boldsymbol{J}^{-1}(\boldsymbol{\eta}) \boldsymbol{s}+\tilde{\lambda}^{T} \dot{\boldsymbol{\Gamma}} \dot{\tilde{\lambda}}
\end{aligned}
$$

If we substitute Eq. (16) into Eq. (20), then the following is obtained:

$$
\dot{V}(t)=-\boldsymbol{s}^{T}\left(\boldsymbol{K}_{d}+\boldsymbol{D}_{\eta}\right) \boldsymbol{s} \leq 0
$$

Eq. (21) shows that the compensation control system is, in general, asymptotically stable. Based on the compensation control law, the schematic diagram for the compensation control system is shown in Fig. 3.

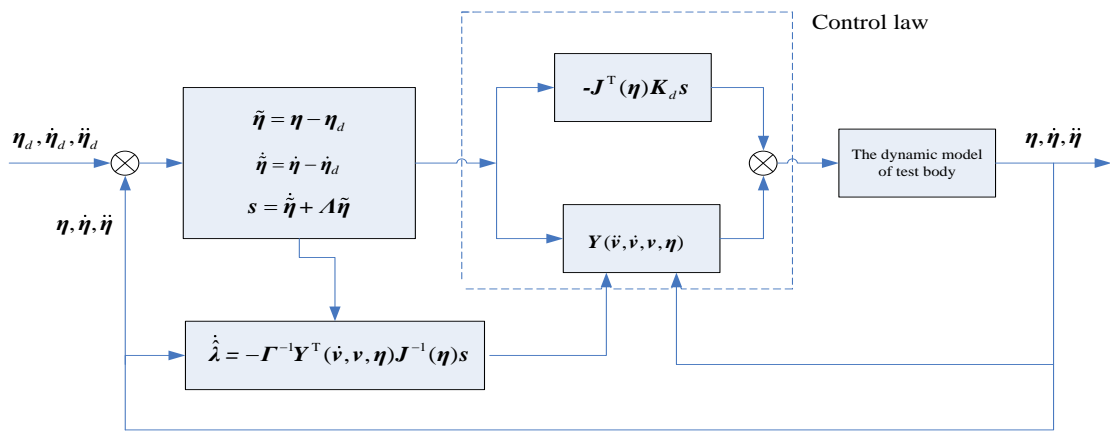

Fig. 3. The schematic diagram for the compensation control system. 


\section{Tests, analysis and results}

Based on the aforementioned method, an experimental verification system has been built in the laboratory at Northwestern Polytechnical University and applied to China's manned space flight engineering, as shown in Fig. $4^{[1]}$.

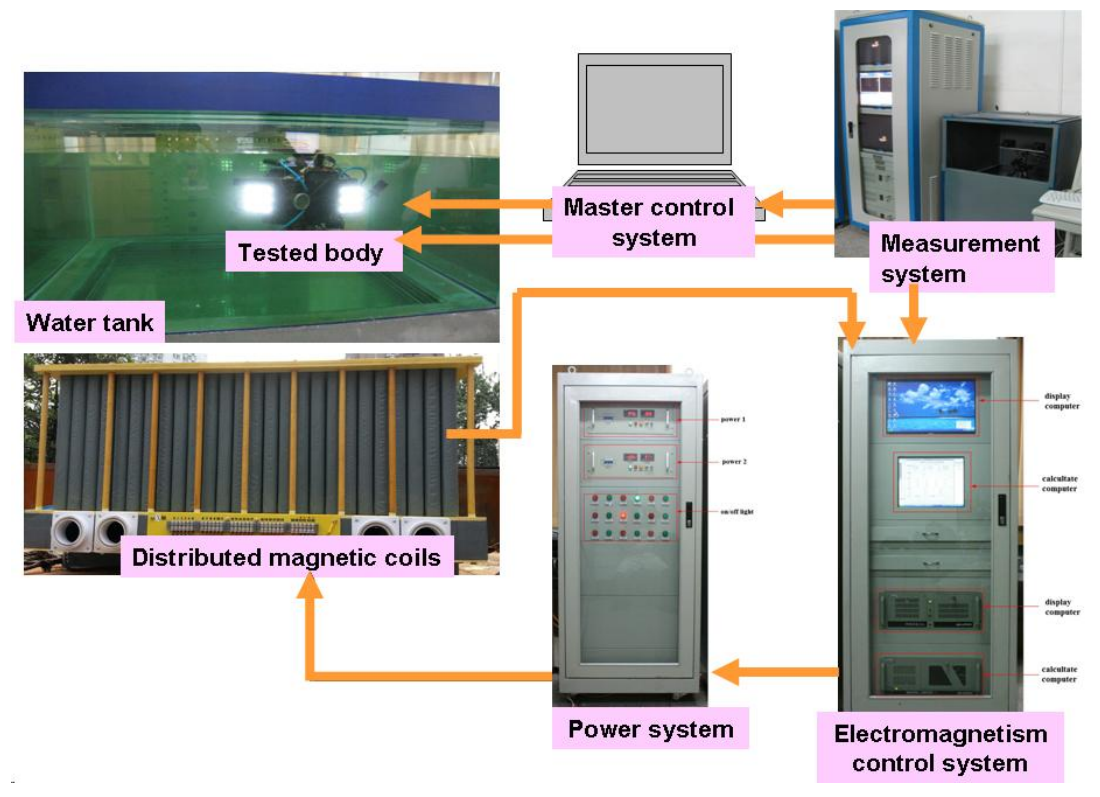

Fig. 4. The composition of the experimental verification system. ${ }^{[1]}$

The experimental verification system has two hybrid test regions, whose areas are $2.5 \mathrm{~m} \times 2.5$ $\mathrm{m} \times 1.5 \mathrm{~m}$ and $24 \mathrm{~m} \times 20 \mathrm{~m} \times 9 \mathrm{~m}$ respectively. The test body used is a polyhedron with 26 surfaces. Six propeller thrusters, categorized in three groups, are symmetrically mounted along three axes of the test body respectively. Its buoyancy reaches approximately $101 \% \mathrm{G}$ (in this case, the buoyancy is bigger than gravity) after being balanced by the liquid-float system, where the electromagnetic force accounts for about $1 \%$ of gravity. The distributed electromagnetic system adopted here has 26 columns $\times 26$ rows. After adjusting the direction of direct current, electromagnetic suction is generated and used for accurate balance. After adjusting the size of electromagnetic force, the test body is able to stay stably suspended at a given point.

\subsection{Rapidly testing the balance of neutral buoyancy state}

In order to verify the micro-gravity level after stable suspension, experiments have been conducted in horizontal plane at several different depths. The electromagnetic force closed-loop control is applied to these depths until the test body gets stable, and then the values of current and voltage are recorded and kept constant. After this, the microgravity level is measured with the z-directional acceleration of the test body (parallel to the local gravitational acceleration) by a standard accelerometer. The whole balancing process is implemented by computers, the time cost for reach the balance state is less than one minute. The results on the plane with the depth of $810 \mathrm{~mm}$ are described with the curve in Fig. 5, and the results on changing depth is described with the curve in Fig. 6. 


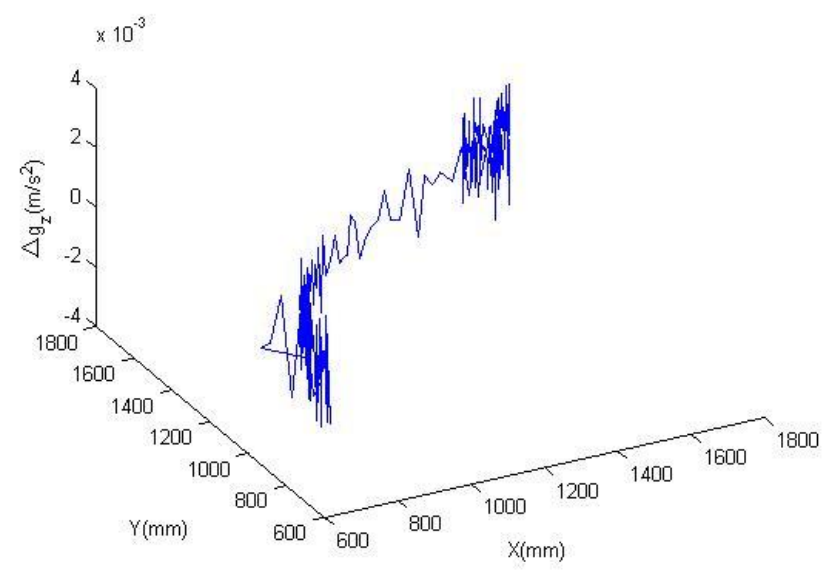

Fig. 5. The test results on the plane with the same depth.

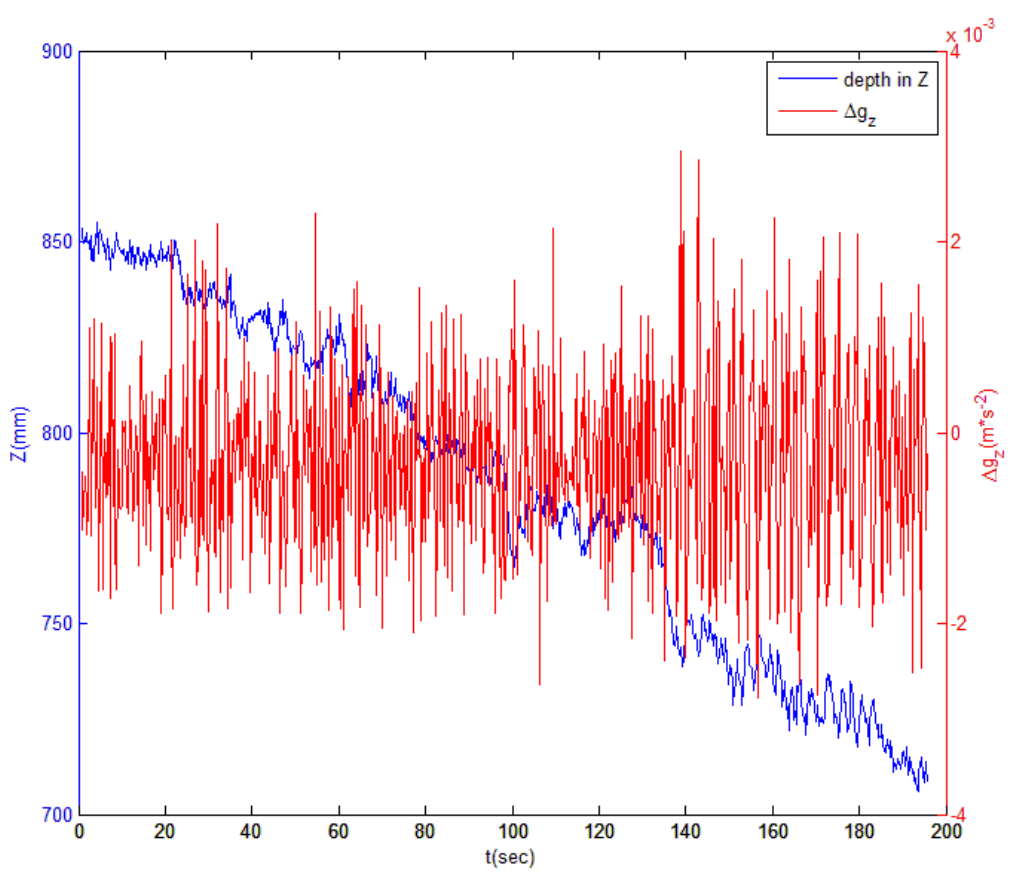

Fig. 6. The test results on changing depth.

As shown in Fig. 5 and Fig. 6, the microgravity level of our experimental verification system reaches up to $10^{-4} \mathrm{~g}$ ( $g$ is the local gravitational acceleration, in above test, $g=9.7836 \mathrm{~m} / \mathrm{s}^{2}$ ). From the above two figures one can infer that the electromagnetic force can be kept constant in the whole experimental space. Also as previously presented, the buoyancy is approximately $101 \% \mathrm{G}$; therefore the microgravity level obtained with the neutral buoyancy method is only $10^{-2} \mathrm{~g}$. Compared with $10^{-4} \mathrm{~g}$ obtained with our system, it appears that the microgravity level obtained with the improving method in this paper is two orders of magnitude higher than the neutral buoyancy method alone.

\subsection{Resistance effect compensation test}


Given the desired ideal states of the test body's motion as described in Eq. (22), which is a uniformly accelerated motion, the pattern of change in linear velocity and angular velocity are described with the following functions in Eq. (22):

$$
\left\{\begin{array}{l}
u=0.004 \mathrm{~m} / \mathrm{s} \\
v=0.004 \mathrm{~m} / \mathrm{s} \\
w=0.001 \mathrm{~m} / \mathrm{s} \\
p_{d}=q_{d}=r_{d}=0
\end{array}\right.
$$

The desired position of the test body is $(668,668,500) \mathrm{mm}$ and the desired attitude is $(0,0,40)$ deg. With the compensation control law as given in Eq. (16) and the prediction law as given in Eq. (15), we choose the positive definite matrix $\Gamma=0.01 I_{6}, \Lambda=\operatorname{diag}\{0.08,0.08,0.08,1.1,1.1,1.1\}$, and the PD control gain matrix $\boldsymbol{K}_{d}=\operatorname{diag}\{17.6,17.6,17.6,33,99,99\} \quad$ to perform compensation tests. At initial time, the test body is placed on the position $(700.884,428.322,510.3647) \mathrm{mm}$ and its attitude is $(0.202,-0.963,-9.681) \mathrm{deg}$, the test results shown in Fig. 7-Fig. 17.

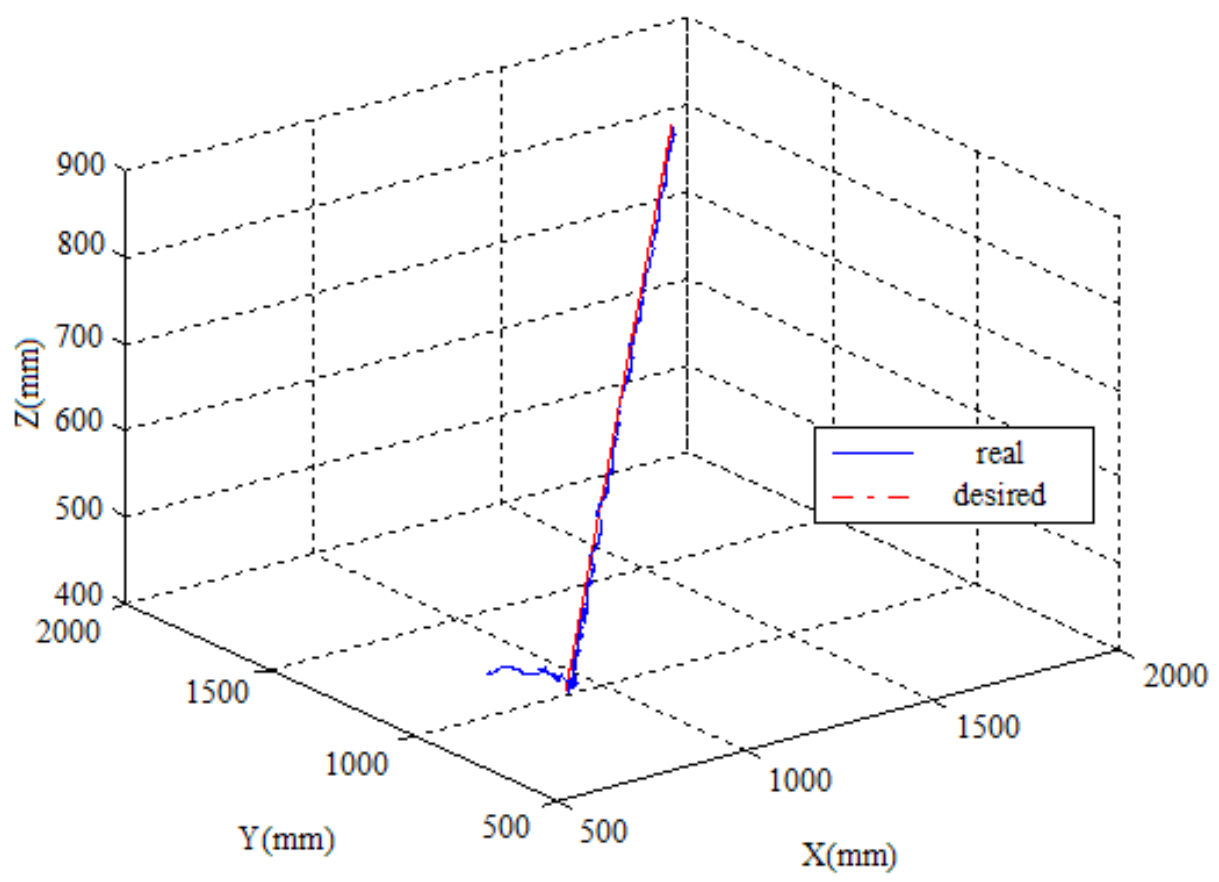

Fig. 7. The trajectory tracking of uniformly accelerated motion. 


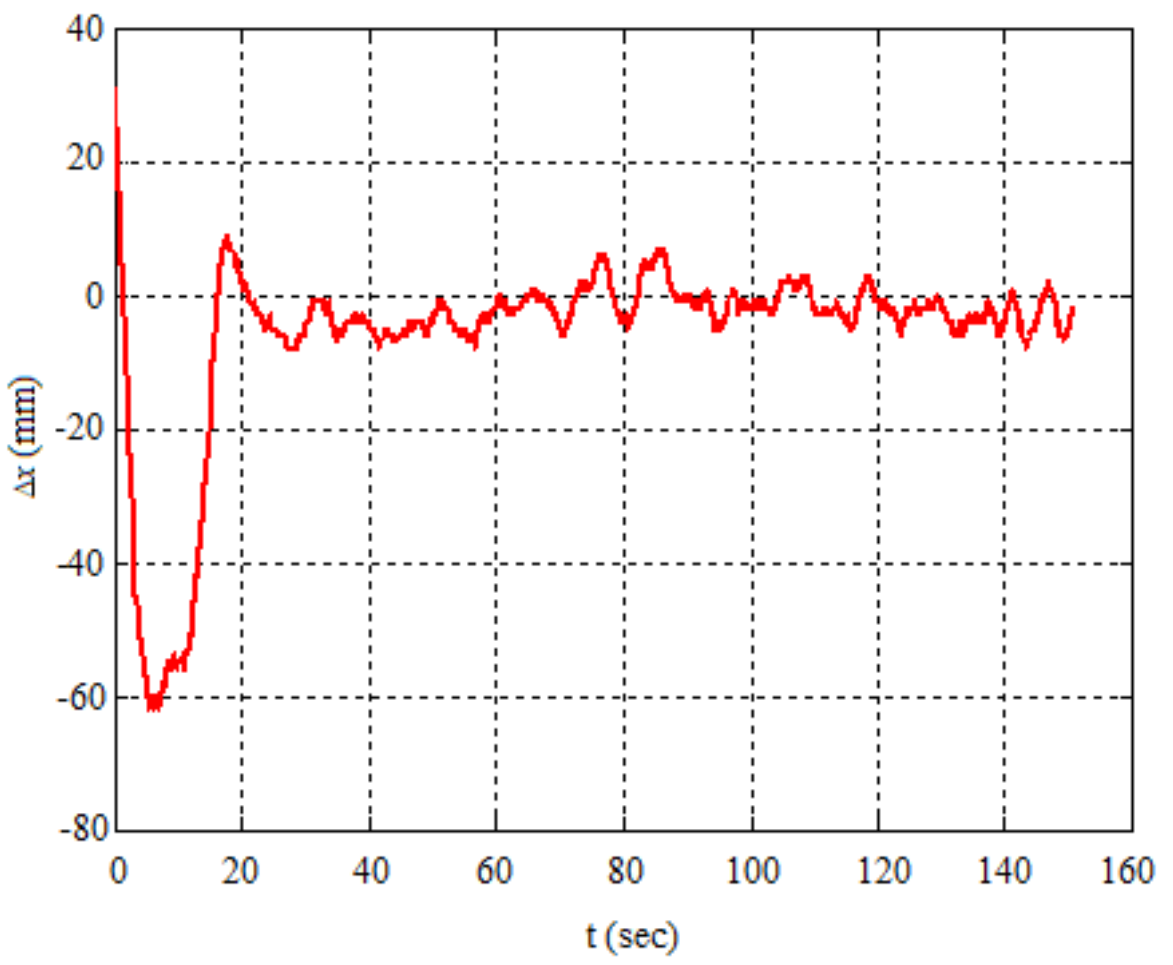

Fig. 8. Position tracking error in X.

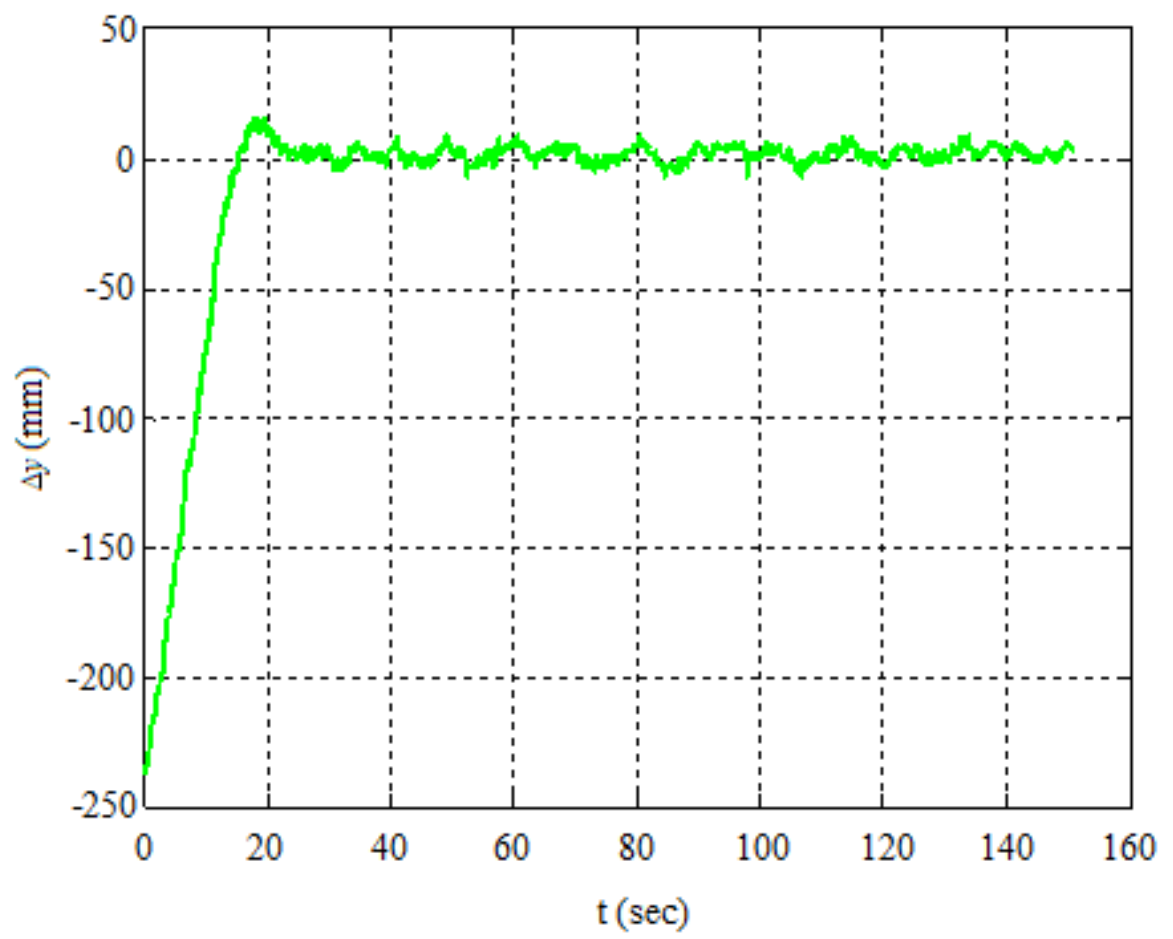

Fig. 9. Position tracking error in Y. 


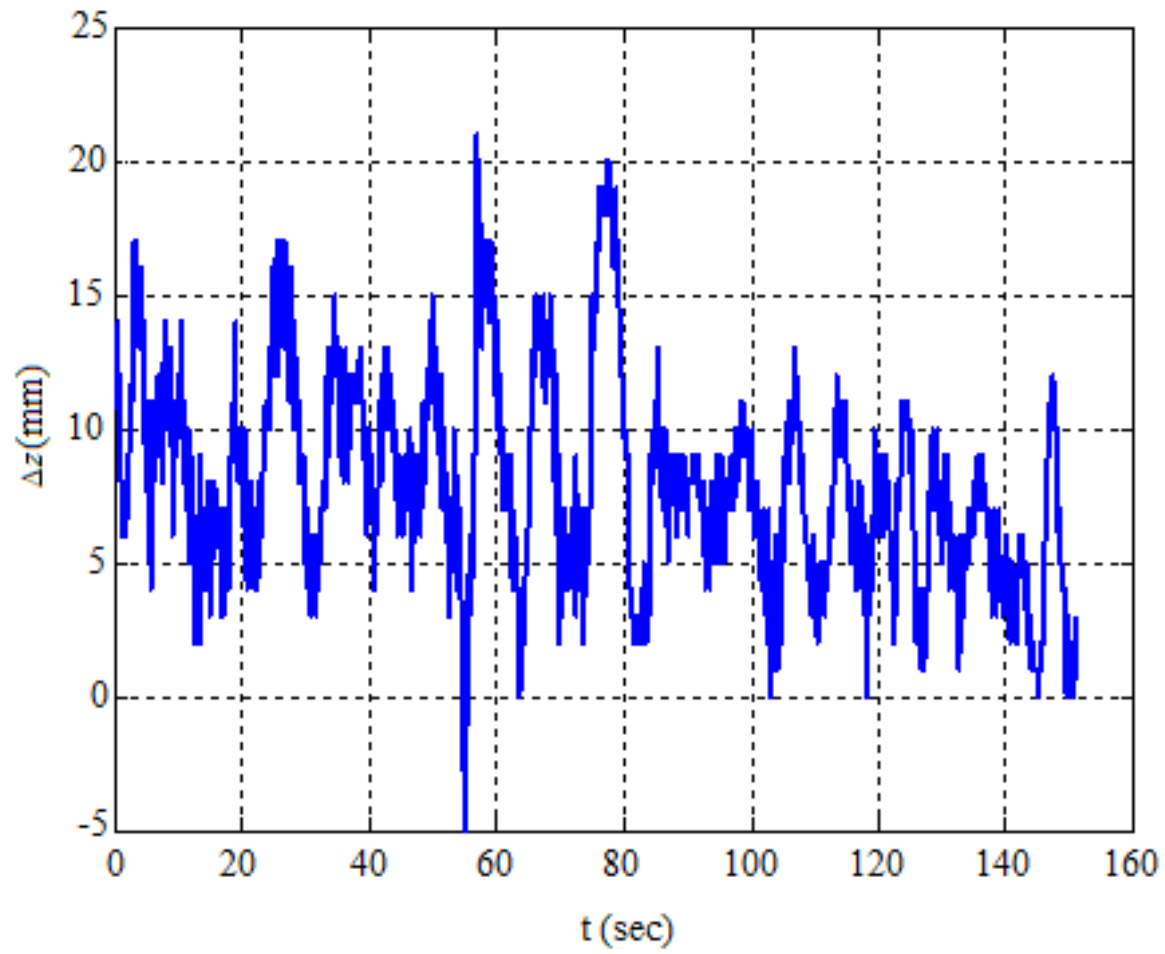

Fig. 10. Position tracking error in $\mathrm{Z}$.

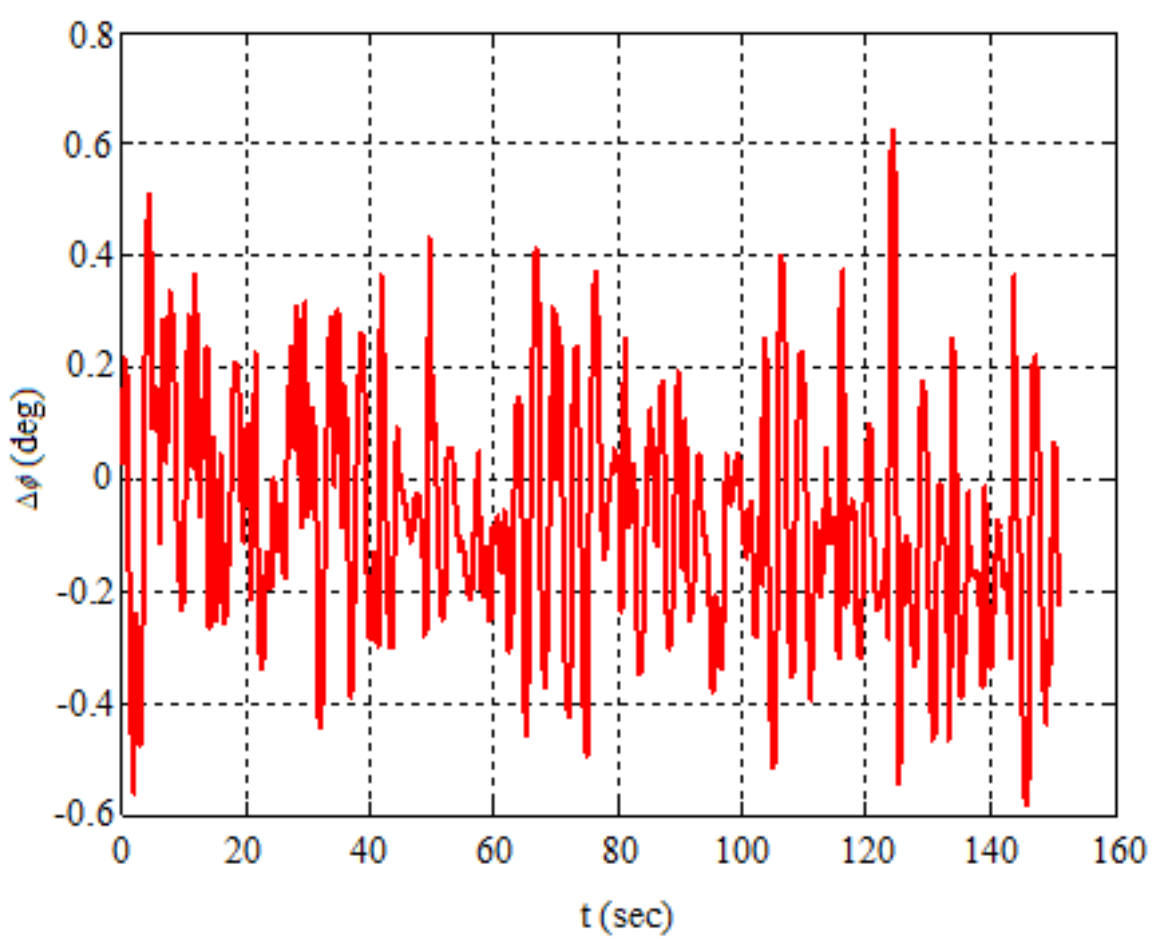

Fig. 11. Attitude tracking error in roll. 


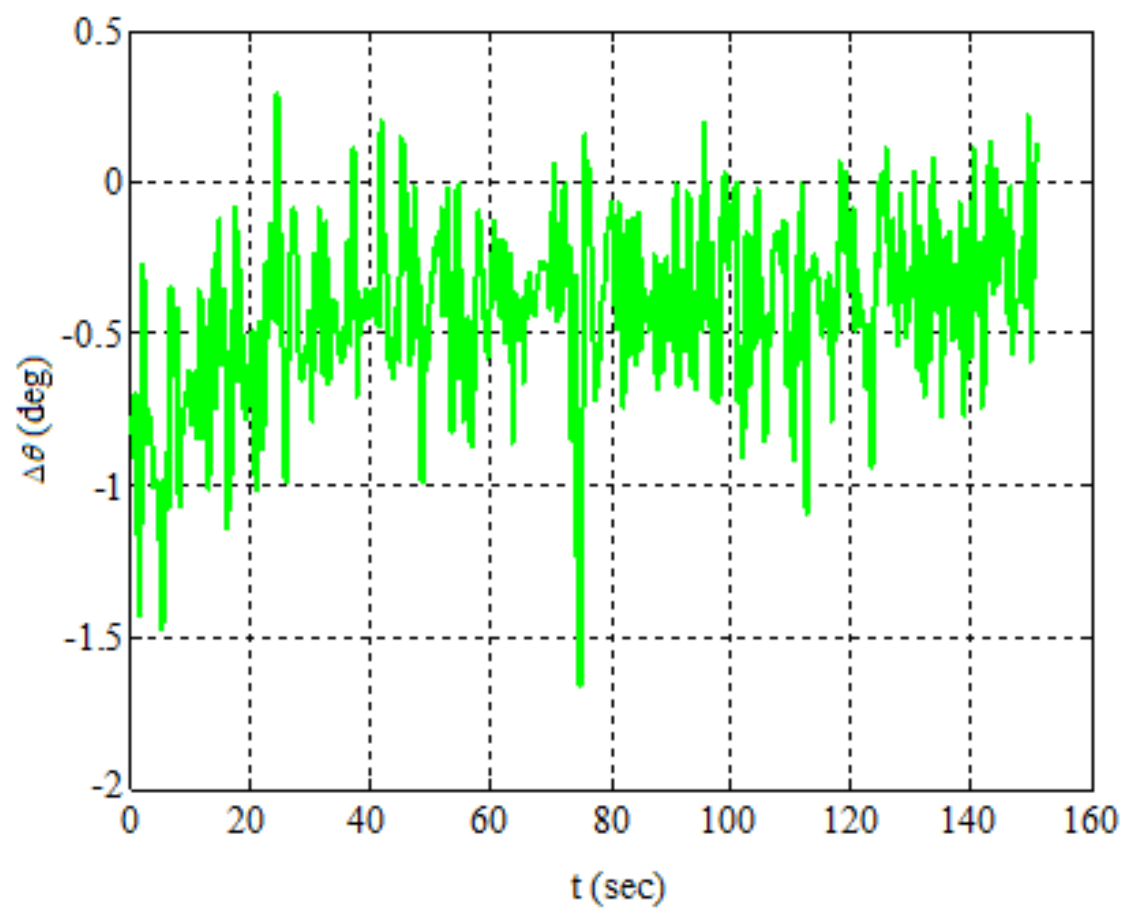

Fig. 12. Attitude tracking error in pitch.

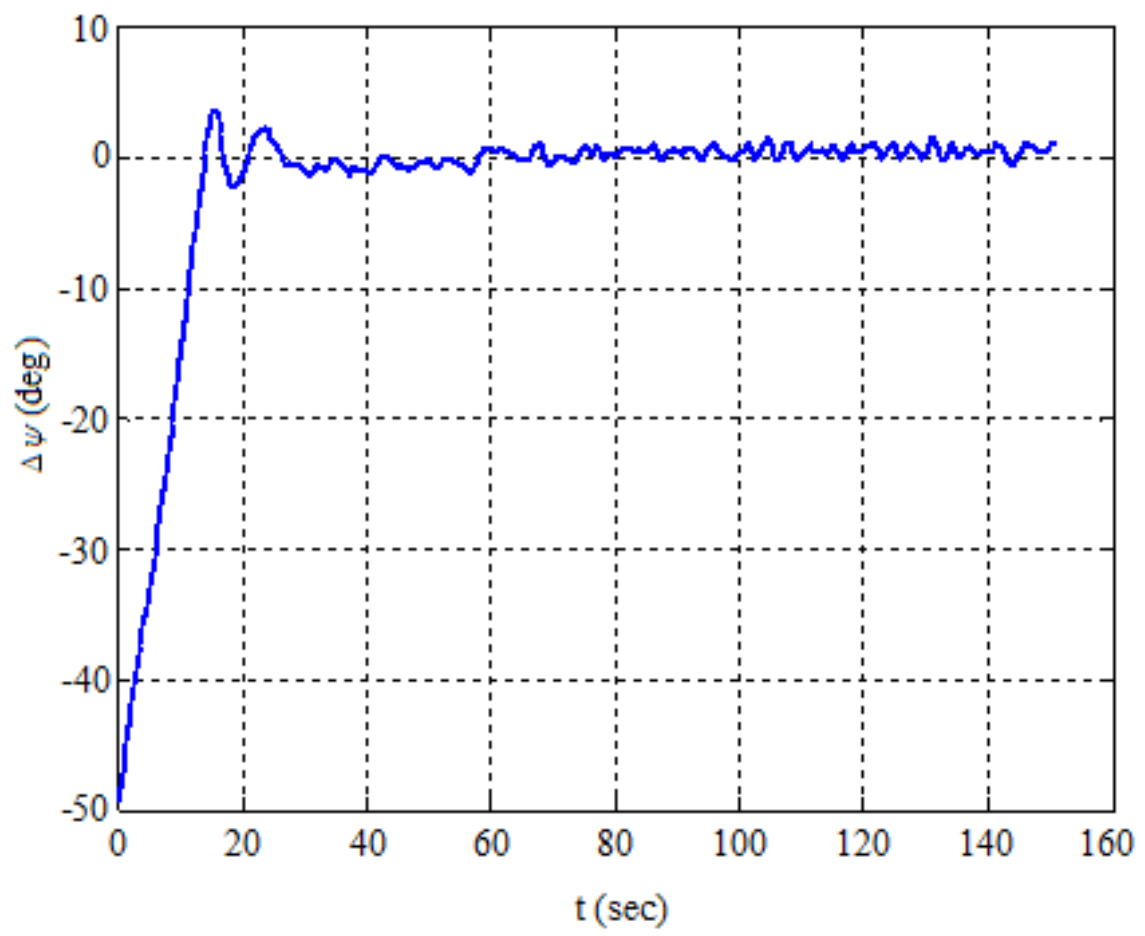

Fig. 13. Attitude tracking error in yaw. 

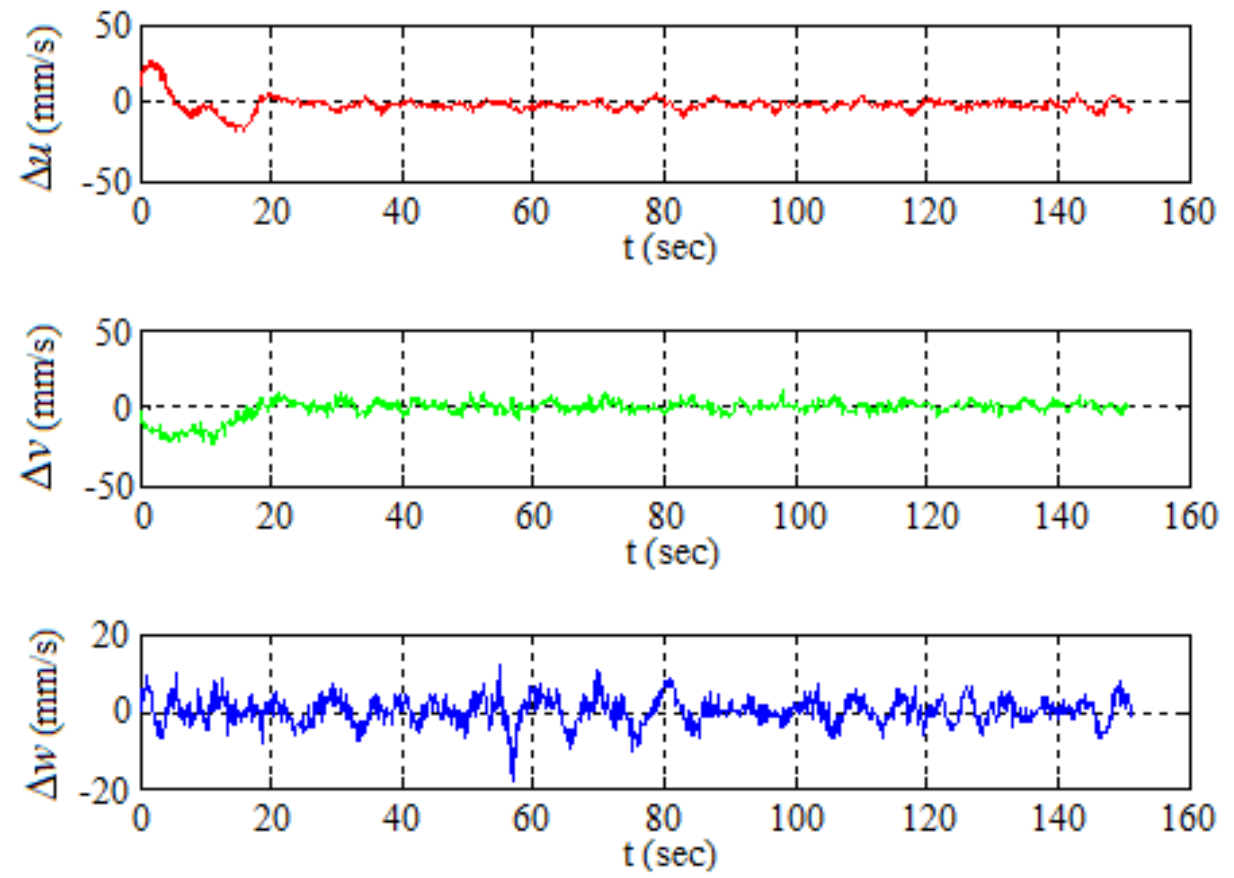

Fig. 14. Linear velocity tracking errors.
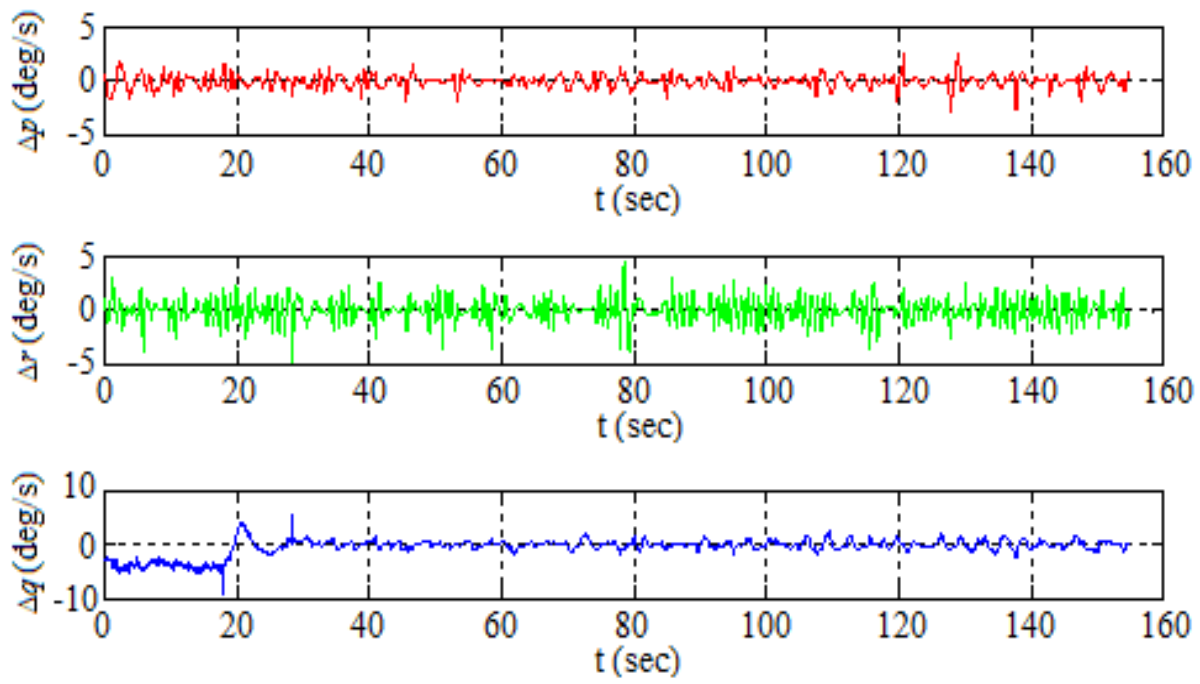

Fig. 15. Attitude angular rate tracking errors. 

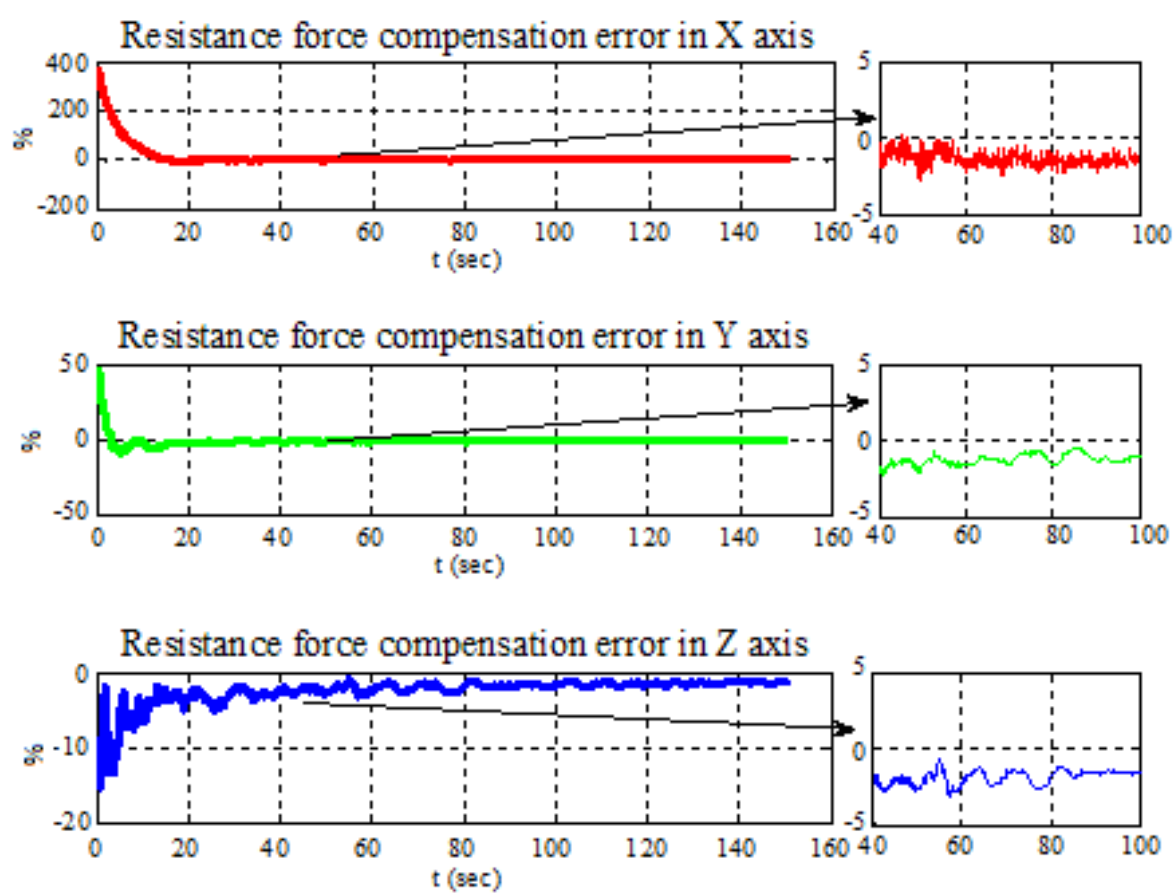

Fig.16. Resistance force compensation errors.
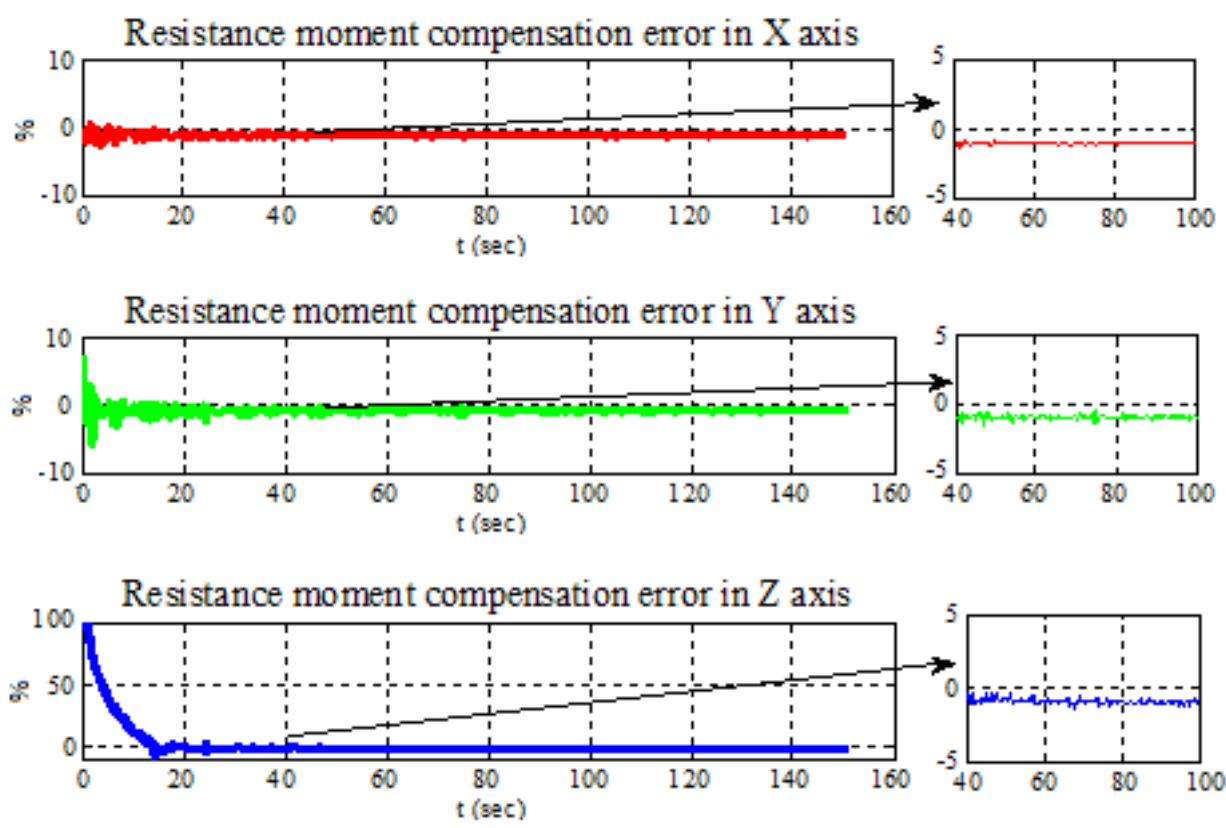

Fig. 17. Resistance moment compensation errors.

The initial errors are large because the test body is not start from the desired condition, it takes about 20 seconds to eliminate those initial errors and reach stable tracking condition. After 20 seconds, from Fig. 8 to Fig. 15, one can see that the position tracking errors are about $\pm 20 \mathrm{~mm}$, the attitude tracking errors are less than $\pm 2^{0}$, the error of linear velocity are less than $\pm 20 \mathrm{~mm} / \mathrm{s}$, the error of attitude angular rate is about $\pm 3^{0} \%$. And from Fig. 16 to Fig. 17, one also can derive that the resistance compensation error is about $2 \%$. The similar demonstration results on tracking circle motions are also obtained. So we can conclude that the test body moves with the desired uniformly 
accelerated motion, and the influence of resistance on motion is offset.

\section{Conclusions}

The neutral buoyancy system is believed to be one of the most suitable micro-G simulation systems on the ground for verifying complex space operations. However, it suffers from two limitations: its complex balancing process, and low fidelity caused by water viscosity. A novel solution is presented to remedy above drawbacks. (i) The GBTB, a special platform developed in this paper, can be used to carry a test body, realize the effect of neutral buoyancy with controllable electromagnetic force, without any additional weight or foam fixed on the test body. Particularly, the GBTB can be used for any new test body, whatever its shape or size is, and not only implement quickly absolute suspension of the test body, but also make the simulation accuracy controllable in real time. Through magnetism-buoyancy hybrid micro-G experiments and tests, the GBTB is proved feasible and effective, and the micro-G simulation accuracy is improved by two orders of magnitude comparing with the conventional neutral buoyancy system. (ii) Considering the test fidelity influenced by water resistance, a compensation system to equalize the water resistance effect during maneuver is developed. A compensation control law and a novel water resistance prediction algorithm are designed by introducing control errors and error rates. The proposed approach is validated by using the magnetism-buoyancy hybrid micro-G simulation system, and the test results show that the resistance compensation error of the system is less than $2 \%$ of the resistance force. Based on the GBTB platform and the compensation system, more elaborate complex space operations will be simulated and conducted in the near future.

\section{Acknowledgements}

The valuable comments from the anonymous reviewer are highly appreciated. This study is financially supported by the Chinese National Science Foundation (Grant No. 11572248, 11472213, 11502203).

\section{References}

[1] Jianping Yuan, Zhanxia Zhu, Zhenfeng Ming, Qiuyue Luo, An innovative method for simulating microgravity effects through combining electromagnetic force and buoyancy, Advances in Space Research, 56(2),2015: 355-364

[2] Ella Marie Atkins, Design and Implementation of a Multiprocessor System for Position and Attitude Control of an Underwater Robotic Vehicle, Thesis for the degree of Master of Science, University of Maryland,1990

[3] Joseph Douglas Graves, Design and Control of a Vehicle for Neutral Buoyancy Simulation of Space Operations. Thesis for the degree of Master of Science, University of Maryland, 1997

[4] Mike, Nick et al. "Toward Autonomous Sampling and Servicing with the Ranger Dexterous Manipulator", 2005: AIAA 2005-6916

[5] Catharine L. R. McGhan, Rebecca L. Besser, Robert M. Sanner, and Ella M. Atkins, 
Semi-Autonomous Inspection with a Neutral Buoyancy Free-Flyer, AIAA Guidance, Navigation, and Control Conference and Exhibit, 21-24 August, 2006, Keystone, Colorado, AIAA 2006-6800

[6] Evelyn Ellis Vance, Adaptive Control of Free-Floating and Free-Flying Robotic Manipulators, Thesis for the degree of Doctor of Philosophy, University of Maryland, 1998

[7] Fossen T I. Guidance and control of ocean vehicles. New York, 1994 